\title{
L'origine ontogénique des racines, puis des bourgeons, néoformes sur des chimères de poi- rier (Pyrus communis L.) et de pommier (Malus pumila Mill.)
}

Luc DECOURTYE

I.N.R.A., Station d'Arboriculture Fruitière, Centre de Recherches d'Angers, Beaucouzé, 49000 Angers

\begin{abstract}
Treize varietés de poirier et deux variétés de pommier, manifestant sur leurs différents organes une constitution en chimère péricline de nature chlorophyllienne ou anthocyanée, ont été mises à bouturer sous brouillard, en comparaison avec leur variété parentale non panachée.

La plupart de ces variétés ont émis des racines dont nous avons cherché à identifier le phénotype en obtenant des pousses feuillées. C'est donc le résultat simplificateur de deux néoformations successives, celle de racines, puis celle des bourgeons, que nous avons pu observer.

Aucune des pousses issues de néoformation ne reproduit la chimère. La plupart d'entr'elles expriment d'une façon homogène le phénotype des tissus profonds L III de la variété panaclıée. Dans quelques cas, rares, des pousses homogènes expriment entièrement le phénotype de la couche moyenne L II. Toutes les pousses de ce type proviennent toujours d'une même racine, qui ne produit pas d'autres phénotypes. La disjonction de la chimère en L II/L III s'effectue done au moment de la néoformation racinaire, celle-ci se faisant le plus souvent à partir de cellules ayant le phénotype L III, exceptionnellement à partir de cellules ayant le phénotype L II.
\end{abstract}

Mots clés additionnels : Bouturage, néoformation, ontogenèse.

Thirteen pear varieties and two apple varieties, which happen to be periclinal chimeras for chlorophyll deficiency or anthocyanin pigmentation, were rooted under mist, together with their parent variety as control. Most were able to differentiate shoots from these roots so that their phenotype for chlorophyll or anthocyanin pigmentation could be established. None of these neoformed shoots showed a chimeral structure. Most of them appeared to carry the phenotype of the internal layer L III. In a few exceptional cases, the origin of the shoots was the L II layer. One root produced only one type of shoot (L III or L II type), so it can be assumed that dechimerisation happens at the root initiation step. This provides a method other than irradiation to ascertain possible chimeral structure and obtain homogeneous shoots.

Additional key words : Rooting, neoformation, ontogenesis.

\section{INTRODUCTION}

Les collections de la Station d'Arboriculture Fruitière d'Angers comportent, principalement en poirier, des variétés panachées d'origine très ancienne, dont on possède également les homologues parentaux homogènes. Ces variétés, panachées tant sur la feuille, le fruit (Fig. 1) ou les jeunes rameaux s'interprètent comme des chimères périclines comportant une déficience chlorophylienne ou anthocyanée dans leurs couches profondes. La conception que l'on se fait du fonctionnement d'un méristème végétatif d'une plante ligneuse selon trois couches imbriquées, relativement indépendantes, a été résumée par ailleurs (DECOURTYE, 1984). L'effet de déchimérisation provoqué par un rayonnement gamma, démontré par P. DoMmerGUES (1961) sur la chimère anthocyanée de Max Red Bartlett, a pu être confirmé sur l'ensemble de ces variétés panachées (DECOURTYE, 1963 et 1969).

Il nous est apparu que ces chimères pouvaient être utilisées pour apporter des informations sur les contri- 


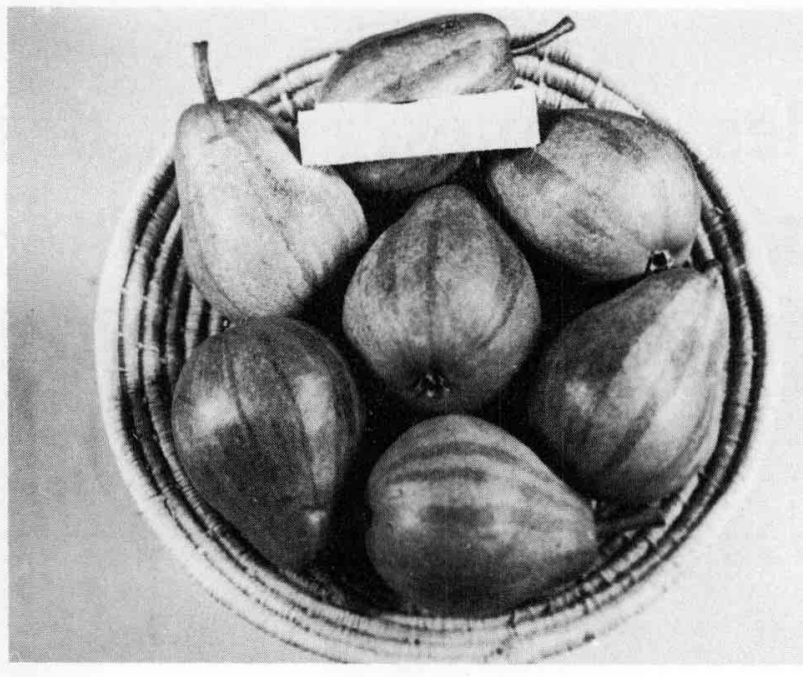

Figure 1

Striations caractéristiques des fruits d'une variété de poire panachée.

Typical stripes on the fruits of a variegated pear cultivar.

Il s'agit ici du clone P 157I, réarrangement ontogénique obtenu après irradiation de Max Red Bartlett, selon la séquence Vert-VertRouge. Les secteurs rouges représentent des affleurements superficiels de la couche L III, qui peut ainsi, à la lumière, exprimer son phénotype. Pour les variétés panachées de nature chlorophyllienne (Vert-Vert-Deficient), ces secteurs d'affleurements de la L III apparaissent au contraire comme des zones plus claires.

The picture shows fruits of clone $P$ 1571, resulting from irradiation of Max Red Bartlett, with a new ontogenetic layer arrangement : Green-Green-Red. The dark sectors are the areas where the contribution of the L III to the fruit flesh comes close to the epidermis, so that the gene for red color receives enough light for its expression. For variegated varieties, which are usually chlorophyll chimeras in the sequence Green-Green-Deficient, the narrow sectors where L III comes close to the surface appear as a paler green color.

butions des deux principales couches L II et L III dans la formation des différents tissus des rameaux. L'essentiel des connaissances actuelles en ce domaine a été obtenu à partir de travaux sur des chimères polyploïdes (DERMEN, 1951 et 1953). Elles avaient l'avantage de permettre un marquage cellulaire facilement reconnaissable in situ. Mais de telles chimères font coexister des cellules qui diffèrent par un génome complet, elles apparaissent rarement spontanément et ne se maintiennent pas. Au contraire les chimères chlorophylliennes et - à un degré moindre anthocyanées sont un phénomène courant pour les plantes ligneuses à multiplication végétative, non seulement les arbres fruitiers mais beaucoup d'espèces ornementales. On peut penser que les deux tissus constitutifs de la chimère ne se distinguent alors que par une différence génétique mineure. Pour deux variétés de poirier utilisées ici (Louise Bonne panachée et Comice panachée) le caractère de déficience chlorophylienne a pu être reconnu d'ordre cytoplasmique (Decourtye, 1973) tandis que le caractère anthocyané de Max Red Bartlett est à contrôle génique, monofactoriel et dominant.

L'organisation ontogénique de telles chimères nous paraît donc plus proche de celle d'un méristème homogène et, par-là, capable d'en reproduire plus fidèlement son fonctionnement. Par contre, le mar- quage chlorophyllien ou anthocyané ne s'exprime pas dans tous les tissus du végétal. Pour révéler le génotype des couches profondes, il nous fallait donc soit obtenir des bourgeons d'origine interne directement sur la tige, ce qui s'est révélé difficile, soit procéder par une double étape, à savoir la néoformation de racines suivie d'une néoformation de bourgeons. Cette méthode, un peu longue, apporte en plus une information sur la zone de différenciation des racines néoformées.

D'un point de vue pratique, nous étions également intéressés par une méthode de séparation des tissus constitutifs d'une chimère qui ne fasse pas appel à l'irradiation. En effet, si cette dernière méthode est efficace, elle est toujours susceptible de provoquer aussi des mutations; ce risque nécessite une longue expérimentation du matériel qui en découle avant de pouvoir le diffuser commercialement.

\section{MATÉRIEL ET MÉTHODES}

Dix variétés de poirier panachées, dont 2 sont synonymes (Beurré d'Arenberg = Beurré d'Hardenpont), ont été bouturées sous brouillard, avec leurs homologues parentaux non panachées, selon la technique décrite par ThiBAULT \& HERMANN (1966) pour la variété Williams. Deux cultivars de pommier, la variété Sikler, sans homologue homogène, et un mutant de porte-greffe de pommier MM 112, précédemment étudié (DECOURTYE, 1963) présentent la même constitution histogénique. Tous ces clones se caractérisent par une déficience chlorophyllienne au niveau de la couche L III (que nous symboliserons par la formule Vert-Vert-Déficient). Cette déficience chlorophyllienne peut présenter différents degrés, d'un blanc pur (albina) pour Louise Bonne panachée par exemple ou Sikler, ou bien un jaune plus ou moins soutenu (xantha) comme pour Comice panachée. Des chimères anthocyanées, dérivées de la variété Williams, ont également été utilisées : la plus connue est le cultivar Max Red Bartlett, mutant anthocyané spontané, dont les tissus profonds sont restés du phénotype Williams (soit Rouge-Rouge-Vert). Deux autres réarrangements chimériques, obtenus par DoMMERGUES (1961) après irradiation de Max Red Bartlett, ont également été bouturés : P 1571 et P 1572. Le premier (Fig. 1) se présente avec des fruits striés de rouge et des feuilles avec une zone centrale anthocyanée, selon les mêmes répartitions que les variétés panachées : il s'interprète comme une chimère péricline de constitution Vert-Vert-Rouge. Le second, P 1572, présente des feuilles vertes à frange périphérique rouge, avec un très fort taux spontané de retour au type vert (58 p. 100). Nous l'interprétons comme une chimère de type Rouge-Vert-Vert. L'absence de fructification de ce clone n'a pas permis de connaître la couleur des fruits.

Trois années de bouturage avec une cinquantaine de boutures par clone et par année ont été nécessaires pour obtenir au moins dix boutures vivantes de chacun de ces clones.

Ces boutures ont été plantées en pleine terre, dans une tranchée drainée et remblayée par un substrat 
horticole de bonne qualité. Les conditions de culture - arrosage, nutrition minérale, désherbage - ont été aussi soignées que possible pour favoriser une bonne croissance.

Après 3 et 4 années de croissance dans cette tranchée, - exceptionnellement 8 années pour quelques clones - des racines ont été séparées de la partie aérienne selon deux modalités. Dans une première expérience, les arbres ont été soigneusement arrachés pour obtenir le maximum de racines. Celles-ci ont alors été sectionnées en tronçons de 20 à $30 \mathrm{~cm}$, placées à plat dans des caissettes, recouvertes de terreau jusqu'à affleurement et placées en serre. Une atmosphère humide était assurée par des arrosages périodiques et un bâchage plastique.

Dans les phases ultérieures, nous avons procédé à un dégagement sur place des racines autour du collet de l'arbre, sectionné celles-ci et retiré non seulement la partie aérienne, mais aussi tout ce qui, dans le sol, pouvait provenir de la bouture originelle. Ces racines sont alors repérées et classées selon leur grosseur en trois catégories (grosses : diamètre supérieur à $30 \mathrm{~mm}$, moyennes, ou fines : diamètre inférieur à $15 \mathrm{~mm}$ ). Le système racinaire complet reste ainsi en place, la coupe des racines affleurant la surface du terreau. L'ensemble est recouvert d'un tunnel plastique pour éviter une accumulation sur la tranchée des eaux de pluie et protéger les jeunes pousses susceptibles d'émerger de ces racines.

Un essai préliminaire a été effectué sur quelques variétés pour déterminer l'influence de la date de sectionnement des racines sur la néoformation de pousses, en procédant de 15 jours en 15 jours depuis la miavril (avant débourrement) jusqu'à la fin de juin. Les amputations de pré-débourrement ont fourni le plus grand nombre de pousses néoformées: c'est donc cette époque qui a été retenue pour l'ensemble des résultats présentés.

Une surveillance mensuelle permet de noter la date d'apparition de nouvelles pousses feuillées, la couleur $\mathrm{du}$ feuillage, et la racine dont elles proviennent.

\section{RÉSULTATS}

Les résultats de bouturage sont irréguliers au cours des différentes campagnes. Certains échecs peuvent être imputés à l'état originel des boutures, ou au bon fonctionnement du système de brumisation, celui-ci devant être en accord avec la climatologie de l'année. Le tableau 1 présente le pourcentage de boutures enracinées et vivantes à la fin de l'automne pour chacun des clones, et ceci pour la meilleure campagne.

On constate que toutes les variétés ont été capables d'assurer la néoformation de racines, avec des fréquences variables de 20 à 90 p. 100, selon les variétés. Il n'apparaît pas par contre que le mutant en chimère ait un comportement systématiquement différent de son homologue homogène.

Si la fréquence d'enracinement des boutures, observée à l'automne de la première année, est dans l'ensemble satisfaisante, il faut souligner qu'une mortalité importante survient au cours de l'hiver, en particulier sur les boutures n'ayant manifesté aucune

\section{TABLEAU}

Pourcentage d'enracinement sous brouillard de 13 variétés de poirier panachées et de deux variétés de pommier, avec leur témoin.

Rooting percentage of soft cuttings from 13 variegated pear varieties and 2 apple varieties, with controls.

\%o de boutures
racinées
(sur
50 boutures)
au moins)

\section{POIRIER}

Chimères chlorophylliennes et leurs témoins

Alexandrine Douillard

$\mathrm{V} V \mathrm{D}$

V V V

82

Beurré d'Amanlis panachée

Beurré d'Amanlis

V V D

Beurré d'Arenberg panachée

$\mathrm{V} V \mathrm{~V}$

\section{2}

Beurré d'Arenberg

$\mathrm{V} V \mathrm{D}$

$\mathrm{V} V \mathrm{~V}$

Beurré d'Hardenpont panachée

Beurré d'Hardenpont

$\mathrm{V}$ V D

$\mathrm{V}$ V

Duchesse d'Angoulême panachée

Duchesse d'Angoulême

V V D

Doyenné du Comice panachée

Doyenné du Comice

$\mathrm{V}$ V

$\mathrm{V} V \mathrm{D}$

V V V

Louise Bonne d'Avranches panachée

Louise Bonne d'Avranches

V V D

$\mathrm{V}$ V V

Président Drouard panachée

Président Drouard

V V D

$\mathrm{V} V \mathrm{~V}$

V V D

V V V

Verte longue d'automne

V V D

66

16

52

60

28

12

64

52

Crassane panachée

Chimères anthocyanées

Max Red Bartlett

Williams

R R V

$\mathrm{V} V \mathrm{~V}$

67

P 1571

$\mathrm{V}$ V R

62

P 1572

R V V

43

45

II POMMIER

Malling Merton 112-5

Malling Merton 112

Sikler

V V D

$\mathrm{V} \mathrm{V}$

V V D

43

70

15 reprise de croissance de la partie aérienne, même lorsque ces boutures ne sont pas transplantées. C'est cette mortalité qui nous a conduit pour certaines variétés à renouveler les campagnes de bouturage pour atteindre au moins 10 boutures vivantes bien constituées par clone. La transplantation en pleine terre d'une bouture de 2 ans ne présente, par contre, pas plus de risque que celle d'un scion fruitier normal, et la croissance ultérieure des plants est satisfaisante. Le comportement agronomique comparé de poirier Williams cultivés sur leurs propres racines a été publié par B. Thibault \& HermanN (1982).

Le tableau 2 résume le comportement de l'ensemble des variétés de poirier et de pommier quant au nombre de plants ayant survécu, le nombre de racines obtenues puis organogènes, le nombre de pousses néoformées et la couleur du feuillage de celles-ci. Ces observations proviennent des plantes cultivées en tranchées et sectionnées sur place. 
Comportement des différentes variétés après sectionnement des racines.

Number of roots and of neoformed shoots, according to phenotype, obtained from the different chimeras of pear and apple.

\begin{tabular}{|c|c|c|c|c|c|c|}
\hline & \multirow[b]{2}{*}{$\begin{array}{l}\text { Nombre de } \\
\text { plants }\end{array}$} & \multirow[b]{2}{*}{$\begin{array}{l}\text { Nombre de } \\
\text { racines }\end{array}$} & \multirow[b]{2}{*}{$\begin{array}{l}\mathrm{Nb} \text { racines } \\
\text { organogènes }\end{array}$} & \multirow[b]{2}{*}{$\begin{array}{l}\text { Nombre de } \\
\text { pousses }\end{array}$} & \multicolumn{2}{|c|}{ Couleur } \\
\hline & & & & & $\begin{array}{l}\text { Chloro- } \\
\text { phyllienne }\end{array}$ & $\begin{array}{l}\text { Déficiente (ou } \\
\text { anthocyanée) }\end{array}$ \\
\hline \multicolumn{7}{|l|}{ I POIRIER } \\
\hline \multicolumn{7}{|l|}{ a) Chimères chlorophylliennes } \\
\hline Alexandrine Douillard panachée & 10 & 37 & 12 & 40 & 9 & 31 \\
\hline Alexandrine Douillard & 10 & 47 & 2 & 9 & 9 & 0 \\
\hline Beurré d'Amanlis panachée & 5 & 10 & 2 & 7 & 3 & 4 \\
\hline Beurré d'Amanlis & 10 & 47 & 14 & 21 & 21 & 0 \\
\hline $\begin{array}{l}\text { Beurré d'Arenberg panachée } \\
\text { Beurré d'Arenberg }\end{array}$ & 2 & 7 & 0 & - & & \\
\hline Beurré d'Harpendont panachée & 9 & 23 & $\begin{array}{l}2 \\
1\end{array}$ & $\frac{3}{2}$ & $\frac{3}{2}$ & $\begin{array}{l}0 \\
0\end{array}$ \\
\hline Beurré d'Hardenpont & - & & & - & & \\
\hline Duchesse panachée & 10 & 33 & 4 & 20 & 20 & 0 \\
\hline Duchesse d'Angoulême & - & & & - & & \\
\hline Doyenné du Comice panachée & 6 & 10 & 0 & - & & \\
\hline Doyenné du Comice & 3 & 7 & 0 & - & & \\
\hline Louise Beurré d'Avranche panachée & 9 & 56 & 42 & 86 & 0 & 86 \\
\hline Louise Bonne d'Avranches & 3 & 19 & 12 & 35 & 35 & 0 \\
\hline Président Drouard panachée & 7 & 15 & 4 & 5 & 0 & 5 \\
\hline Président Drouard & 5 & 15 & 1 & 1 & 1 & 0 \\
\hline Verte longue panachée & 6 & 15 & 6 & 30 & 0 & 30 \\
\hline Verte longue d'automne & 2 & 6 & 2 & 14 & 14 & 0 \\
\hline Crassane panachée & 6 & 11 & 0 & - & & \\
\hline \multicolumn{7}{|l|}{ b) Chimères anthocyanées } \\
\hline \multicolumn{7}{|l|}{$-1^{\text {re }}$ année de bouturage } \\
\hline Max Red Bartlett & 10 & 41 & 16 & 50 & 48 & 2 \\
\hline Williams & 10 & 36 & 15 & 45 & 45 & 0 \\
\hline$-2^{\mathrm{e}}$ année de bouturage & & & & & & \\
\hline Max Red Bartlett & 5 & 35 & 35 & 154 & 154 & 0 \\
\hline P 1571 & 5 & 34 & 18 & 26 & l & 25 \\
\hline P 1572 & 5 & 15 & 14 & 50 & 50 & 0 \\
\hline \multicolumn{7}{|l|}{ II POMMIER } \\
\hline Malling Merton 112-5 & 6 & 54 & 27 & 77 & 9 & 68 \\
\hline Malling Merton 112 & 5 & 62 & 62 & 134 & 134 & 0 \\
\hline Sikler & 5 & 55 & 9 & 16 & 0 & 16 \\
\hline
\end{tabular}

Sur les 24 cultivars de poiriers, 2 n'ont pas survécu (Beurré d'Hardenpont et Duchesse d'Angoulême). Quatre d'entr'eux (Beurré d'Arenberg panaché, Doyenné du Comice panachée ou non, ainsi que Crassane panachée) n'ont pas formé de bourgeons.

Dans tous les autres cas, des pousses néoformées sont apparues tout au long de la saison de végétation, du 15 mai pour les premières au 15 octobre pour les plus tardives. Les cultivars témoins, homogènes dans leurs différentes couches, ont fourni des pousses homogènes, semblables à la variété de départ.

Dans les cas où la variété est en chimère, aucune des pousses obtenues ne reproduit celle-ci. On obtient le plus souvent (plus de 90 p. 100 des cas) des pousses homogènes pour la constitution génétique de la L III, c'est-à-dire déficientes chlorophylliennes pour les variétés panachées ou dépourvues d'anthocyanes pour les chimères anthocyanées. Il y a toutefois des exceptions pour lesquelles on observe aussi le phénotype homogène de la L II. C'est ainsi qu'Alexandrine Douillard panachée fournit 9 pousses chlorophyllien- nes sur 40, Beurré d'Amanlis panachée en fournit 3 sur 7, Max Red Bartlett fournit 2 pousses homogènes rouges sur 50 et $\mathrm{P} 1571$ (V.V.R.) fournit 1 pousse verte sur 26 ; le pommier MM 112-5 (V.V.D.) fournit 9 pousses chlorophylliennes sur 77 , reproduisant ainsi le porte-greffe MM 112 d'origine. Enfin deux variétés panachées de poirier (Beurré d'Hardenpont panachée et Duchesse panachée) ne produisent que des pousses correspondant au phénotype de la couche intermédiaire L II et s'écartent ainsi significativement du comportement général. Toutefois l'effectif de racines émises est trop faible ( 1 pour la première, 4 pour la seconde) pour risquer des hypothèses explicatives.

Une même racine ne fournit qu'un type de pousses, soit du phénotype L II, soit du phénotype L III.

C'est donc au moment de la néoformation de la racine que s'effectue la disjonction entre les deux phénotypes. La néoformation d'une racine à partir de cellules de la couche L II est un phénomène plutôt rare : on en trouve 1 sur 12 pour Alexandrine Douillard panachée, 1 sur 2 pour Beurré d'Amanlis panachée, 1 
sur 51 pour Max Red Bartlett, 1 sur 18 pour $P 1571$ et enfin 5 sur 27 pour le pommier MM 112-5.

D'un point de vue pratique, si les pousses chlorophylliennes obtenues pour Alexandrine Douillard panachée et Beurré d'Amanlis panachée n'ont pas d'intérêt, puisqu'elles reproduisent les variétés de départ, il en va tout autrement des deux pousses homogènes rouges obtenues sur Max Red Bartlett. Cette variété qui a connu une certaine extension en culture (STARK, 1971) présente une instabilité importante en faveur de rameaux homogènes verts, d'autant plus envahissants qu'ils sont plus vigoureux que les rameaux en chimère. Il s'ensuit une taille plus difficile et, à la récolte, une complication des opérations de triage, puisque les fruits rouges et les fruits verts doivent être commercialisés séparément. Les pousses néoformées homogènes rouges échappent donc désormais à cet inconvénient majeur. Elles ont été débarrassées par thermothérapie des viroses qu'elles hébergeaient et sont désormais disponibles pour une diffusion commerciale.

Le tableau 2 montre également qu'une partie seulement des racines sectionnées (35 p. 100) est capable de former des pousses, les racines les plus grosses ayant une production légèrement supérieure. Chacune de ces racines organogènes ne donne naissance qu'à un petit nombre de pousses (en moyenne 2,7). Cette proportion de racines organogènes, ainsi que le nombre moyen de boutures formées dépend des variétés : le groupe Williams offre le meilleur rendement, avec 60 p. 100 de racines organogènes et 3,3 pousses par racine. A l'intérieur de chaque couple variétal, il n'apparaît pas par contre de différence systématique entre un cultivar et son mutant panaché.

Cette double efficacité s'améliore avec l'âge des arbres, comme on peut le constater sur le tableau 3 où on dispose, pour des cultivars du groupe Williams, de 3 âges différents pour l'amputation des racines. La fréquence des racines organogènes augmente sensiblement de 10 p. 100 par année, en même temps que le nombre moyen de pousses par racine double quand on

\section{TABLEAU 3}

Evolution du nombre de racines organogènes et du nombre de pousses néoformées en fonction de l'âge des arbres (cv. Williams).

Number of organogenetic roots and number of neoformed shoots per root, according to age of trees (cv. Williams).

\begin{tabular}{ccccc}
\hline $\begin{array}{c}\text { Age du } \\
\text { plant }\end{array}$ & $\begin{array}{c}\text { Nombre de } \\
\text { plants }\end{array}$ & $\begin{array}{c}\text { Nombre de } \\
\text { racines }\end{array}$ & \multicolumn{2}{c}{$\begin{array}{c}\text { \% de racines } \\
\text { organogenes de pousses }\end{array}$} \\
par racine
\end{tabular}

passe de 4 à 8 ans. Cet effet bénéfique du vieillissement suffit d'ailleurs à rendre compte des résultats inférieurs obtenus sur racines isolées au début de cette étude.

\section{CONCLUSION}

Avec des succès divers, toutes les variétés de poirier ou de pommier utilisées sont apparues capables de néoformations racinaires, bien que ce matériel végétal soit dans un état physiologique parfaitement adulte. Dans tous les cas, les semis à l'origine de ces variétés remontent à plus d'un siècle, et d'innombrables multiplications végétatives par greffage sont intervenues depuis cette époque. La plupart d'entre elles se sont également montrées capables de néoformations caulinaires sur ces racines. Le résultat phénotypique que l'on peut observer additionne les conséquences simplificatrices de ces deux néoformations.

Il se confïme que les racines néoformées proviennent le plus souvent de la couche L III. Quelques-unes proviennent de la couche L II, ce qui est en accord avec la conception des contributions respectives des couches L II et L III à la structure des tiges (C. PRATT, 1983). Le fait que l'on n'ait pas retrouvé de pousses en chimères donne à penser que peu de cellules participent à la néoformation d'une racine. L'observation qu'une même racine ne fournit toujours qu'un seul type de rameau indique que c'est au moment de la néoformation racinaire que s'effectue la déchimérisation.

La méthode ainsi décrite constitue donc un moyen d'étude des variétés en chimère, et permet d'en isoler chacun des phénotypes constitutifs. Elle a donné en tout point des résultats conformes à ceux précédemment obtenus par irradiation. Elle est certes plus longue, mais elle a l'avantage de ne pas cumuler, comme le fait l'irradiation, un effet mutagène avec la déchimérisation. Elle a ainsi permis d'obtenir une souche commerciale de Max Red Bartlett qui échappe aux risques de réversion.

Elle peut constituer une méthode d'étude pour d'autres mutants, importants dans les cultures ornementales ou fruitières, notamment les types «spurs », pour lesquels la structure, chimérique ou non, reste controversée.

Reçu le 16 décembre 1985. Accepté le 25 septembre 1986.

\section{REMERCIEMENTS}

L'auteur remercie A. CORNU \& D. CORNU pour leur contribution appréciée à l'amélioration du manuscrit.

\section{RÉFÉRENCES BIBLIOGRAPHIQUES}

Decourtye L., 1963. Action des radiations ionisantes sur une chimère de pommier. Ann. Amélior. Plantes, 13 (2), 133-140.

Decourtye L., 1969. L'utilisation des rayonnements ionisants pour l'amélioration des arbres fruitiers. Genetica Agraria, Vol. XXIII, Fasc. 1-4, 166-175.
Decourtye L., 1973. Hérédité de quelques caractères marqueurs et possibilités offertes par la mutagenèse. C.R. Symposium I.S.H.S. «Culture du Poirier », Angers, 4-8 Sept. 1972, 93-110.

Decourtye L., 1984. L'obtention de nouvelles variétés en horticulture ornementale. In «Arbres et Arbustes d'ornement des régions 
méditerranéennes », R. Bossard, P. Cuisance, J. B. Baillère Ed., pp. XL-XLVI.

Dermen H., 1951. Ontogeny of tissues in stem and leaf of cytochimeral apples. Am. J. Bot., 38 (10), 753-761.

Dermen H., 1953. Pattern of tetraploidy in the flower and fruit of a cytochemical apple. J. Hered., 44 (1), 30-39.

Dommergues P., 1961. Action des rayons gamma sur les bourgeons de la variété de poirier Max Red Bartlett. Ann. Amélior. Plantes, 11 (3), 349-356.

Pratt C., 1983. Somatic selection and chimeras, pp. 172-185. In
"Methods in Fruit Breeding », Ed. Moore and Janick, Purdue University Press.

Stark P., 1971. Red pears stage a big comeback. Western Fruit Grower, 8-12 December.

Thibault B., Hermann L., 1966. Essais de bouturage herbacé de la variété Williams (Pyrus communis L.). Ann. Amélior. Plantes, 16 (3), 273-298.

Thibault B., Hermann L., 1982. Comportement agronomique de poiriers "Williams " cultivés sur leurs propres racines. Arbo. Fruit., $\mathrm{n}^{\mathrm{os}} 341 / 342,35-36$ 